\title{
A statistical video content recognition method using invariant features on object trajectories
}

\author{
A. Hervieu, P. Bouthemy, and J.-P. Le Cadre
}

\begin{abstract}
This work is dedicated to a statistical trajectorybased approach addressing two issues related to dynamic video content understanding: recognition of events and detection of unexpected events. Appropriate local differential features combining curvature and motion magnitude are defined and robustly computed on the motion trajectories in the image sequence. These features are invariant to image translation, inthe-plane rotation and spatial scaling. The temporal causality of the features is then captured by Hidden Markov Models dedicated to trajectory description, whose states are properly quantized values. The similarity between trajectories is expressed by exploiting this quantization-based HMM framework. Moreover statistical techniques have been developed for parameter estimations. Evaluations of the method have been conducted on several data sets including real trajectories obtained from sport videos, especially Formula One and ski TV program. The novel method compares favorably with other methods including feature histogram comparisons, HMM/GMM modeling and SVM classification.
\end{abstract}

Index Terms-Pattern classification, Video signal processing, Hidden Markov models, Motion analysis.

\section{INTRODUCTION}

This work is motivated by the problem of video motion class detection in order to understand object behaviors. The underlying issue is the content-based exploitation of video footage which is of continuously increasing interest in numerous applications, e.g., for retrieving video sequences in large TV archives [12] [42], creating automatic video summarization of sport TV programs [32], or detecting specific actions or activities in video-surveillance [6] [26]. It implies to shorten the well-known semantic gap between computed low-level features and high-level concepts. Considering 2D trajectories is attractive since they form computable image features which capture elaborated spatiotemporal information on the viewed actions. Methods for tracking moving objects in an image sequence are now available to get reliable enough 2D trajectories in various situations. These trajectories are given as a set of consecutive positions in the image plane $(x, y)$ over time. If they are embedded in an appropriate modeling framework, highlevel information on the dynamic scene can then be reachable.

The developed method aims at designing a general trajectory classification method taking into account both the trajectory shape (geometrical information related to the type

A. Hervieu, P. Bouthemy and J.-P. Le Cadre are with the INRIA, Centre Rennes - Bretagne Atlantique, Campus Universitaire de Beaulieu, 35042 Rennes Cedex, France (ahervieu@irisa.fr, bouthemy@irisa.fr and lecadre@irisa.fr).

Copyright (c) 2008 IEEE. Personal use of this material is permitted. However, permission to use this material for any other purposes must be obtained from the IEEE by sending an email to pubs-permissions@ieee.org. of motion and to variations in the motion direction) and the speed changes of the moving object on its trajectory (dynamics-related information). Unless required by a specific application, the process should not be affected by the location of the trajectory in the image plane (invariance to translation), by its direction in the image plane (invariance to rotation) and by the distance of the viewed action to the camera (invariance to scale). A robust enough framework is also desired since local differential features computed on the extracted trajectories are prone to be noise corrupted. The method should not exploit strong a priori information on the scene structure, the camera set-up, the 3D object motions.

This paper tackles two important tasks related to dynamic video content understanding within a unique trajectory-based framework. The first considered problem is recognizing (or retrieving) events in videos. Semantic classes of dynamic video contents are learned from a set of representative training trajectories and candidate trajectories are assigned to the most relevant (nearest) class. The second task is detecting unexpected events by comparing the test trajectories to representative trajectories of known classes of events.

The remainder of the paper is organized as follows. In Section 2, related works on trajectory-based video content analysis are outlined. In Section 3, the considered local differential features to represent 2D trajectories are introduced. It is shown that they are invariant to $2 \mathrm{D}$ translation, $2 \mathrm{D}$ rotation and scale transformation, and their computation is also described. Section 4 presents the developed HMMbased framework to model trajectories. It can be viewed as a (statistical) quantization of the local features while accounting for their temporal evolution. A way to select the number of states when considering this quantization framework is also proposed, as well as the HMM-based similarity measure used to compare or to classify trajectories. Section 5 deals with the video understanding tasks: event retrieval and detection of unexpected events. Section 6 introduces other classification methods which will intervene in the comparative experimental evaluation of the proposed method. In Section 7, the two data sets (composed of trajectories computed in sport videos) used to test and compare the methods are presented. Results are reported and discussed. Concluding remarks are then given.

\section{RELATED WORK}

Trajectory analysis can help recognizing events, actions, or interactions between people and objects. Several methods have been developed to compare and classify trajectories in order to analyze the content of video sequences. Two steps are needed 
to consider and compare trajectories. First, an efficient representation, able to characterize trajectory properties, is needed. In the video context, for general use, some relevant invariances have to be considered. Then, for comparison purpose, these informations shall be modeled precisely, keeping the important spatio-temporal semantics of the trajectories. In this section, a brief overview of these two aspects of the trajectory analysis is presented.

\section{A. Trajectory feature representation}

Various methods consider different features (and mixture of feature) to process video trajectories, some first considering point coordinates [36] [35] [4] [16] [9] [1] [2] [41] [37] and local orientations [43] [33] on image trajectories as input features. Using these features leads to express strict spatial similarity between trajectories. Other techniques use velocities [43] [48] and curvatures [30] [34] as features to compare 2D trajectories, but visual velocity and curvature still depend on the distance of the viewed action to the camera. In [13], Fashandi et al. consider a rotation invariant representation using relative sequences of angles. In combination with some features defined above, some recent relevant works deal with the color density (RGB information) [25] [26] and the sizes of the tracked objects [28] [27].

All the aforementioned methods exploit features that are not at the same time invariant to translation, rotation and scale transformation. A work by Bashir et al. [3] defined a feature representation invariant to translation and rotation, where scaling effects can be handled by resampling the trajectory data to a common sample size. In this paper, the rough video trajectory information is considered by defining a relevant feature, keeping the motion and shape aspects while standing directly invariant to relevant transformations (including rotation, translation and scaling).

\section{B. Trajectory modeling}

In the past few years, several modelings dealing with the spatio-temporal similarities between considered trajectories have been proposed. Johnson and Hogg [29] described a method representing the trajectory distributions, using flow vectors to train some competitive neural networks. Buzan et al. [8] resorted to the Longest Common Subsequence (LCSS) distance [47] to classify trajectories computed in an image sequence acquired by a single stationary camera for video surveillance. This method is based on a hierarchical unsupervised clustering of trajectories where trajectory features are vectors of $2 \mathrm{D}$ coordinates of the trajectory points. Wang et al. [48] introduced a novel similarity measure based on a modified Hausdorff distance and a comparison confidence measure. Bashir et al. presented a trajectory-based real-time indexing method [4] segmenting the trajectories in subtrajectories using curvature informations, and applying PCA (principal components analysis) and spectral clustering. A system that learns patterns of activity from trajectories and hierarchically classifies sequences using a codebook was developed by Stauffer and Grimson [46]. Remagnino et al. [45] proposed an agent architecture for multi-camera behavior classification in visual surveillance. Some last work used automatically defined grammar rules to model the basic motion patterns of moving objects, using a minimum description length principle [50].

Recent works mainly explored modeling frameworks such as PN (Probabilistic Network) [38] [31] [24] [36] [17] [11], HMM (Hidden Markov Model) [34] [5] [10] [39] or SMC (Semi-Markov Chain) [23] to efficiently express the temporal information (causality) embedded in video trajectories and the semantic meaning that they convey. Porikli defined relevant distances to handle trajectories, especially a HMM-based distance using a Rabiner distance between HMMs to compare trajectories [43]. A comparison of similarity measures relying on some of these modeling can be found in [49]. Nevertheless, the methods based on HMMs, SMCs or DPNs developed so far need much data to efficiently model feature distributions so that these methods are unadapted to treat short trajectories (see subsection 4.1).

The novel method developed in this paper considers the trajectory as a dynamical pattern where the other modelings described above consider the trajectories as attached to the precise filmed scene architecture (for example, paths modeling in a parking video systems). So the approach designed here is different from those proposed so far in several points. First, local differential trajectory features which are able to jointly capture information on the trajectory shape and on the object speed are introduced. Besides, they are inherently invariant to translation, rotation and scale transformations. A procedure to compute them, efficient and robust to noise, has also been developed. Second, temporal evolution of these features over the trajectory curve is explicitly accounted for by considering an original and effective HMM scheme. Indeed, the HMMs states are given by properly quantizing the real feature values. This HMM method is also able to process trajectories of any sizes (especially small trajectories), and efficient HMM parameter estimations are proposed. Moreover, a HMM distance which can be exploited both for recognizing dynamic video contents and detecting unexpected events has been adopted. All these elements make the overall proposed framework automatic, general and flexible.

\section{INVARIANT LOCAL TRAJECTORY FEATURES}

A feature that represents both trajectory shape and object acceleration (more specifically, velocity magnitude change is meant) is required to capture the full intrinsic properties of a video trajectory. As stressed in the introduction, it should also be invariant to $2 \mathrm{D}$ translation, $2 \mathrm{D}$ rotation and scale transformation which will be helpful in most video applications, and may allow comparison of trajectories from different cameras.

\section{A. Trajectory kernel smoothing}

A trajectory $T_{k}$ is defined by a set of $n_{k}$ points $\left\{\left(x_{1}, y_{1}\right), . .,\left(x_{n_{k}}, y_{n_{k}}\right)\right\}$ corresponding to the successive image positions of the tracked object in the image sequence (video shot). The term "object" must be understood in a broad sense, i.e., interest point, gravity center of a segmented region, window center,... To reliably compute the local differential trajectory features, a continuous representation of the curve 
formed by the trajectory is needed. To this end, a kernel approximation of $T_{k}$ is performed, defined by

$$
\hat{u}_{t, h}=\frac{\sum_{j=1}^{n_{k}} e^{-\left(\frac{t-j}{h}\right)^{2}} x_{j}}{\sum_{j=1}^{n_{k}} e^{-\left(\frac{t-j}{h}\right)^{2}}}, \hat{v}_{t, h}=\frac{\sum_{j=1}^{n_{k}} e^{-\left(\frac{t-j}{h}\right)^{2}} y_{j}}{\sum_{j=1}^{n_{k}} e^{-\left(\frac{t-j}{h}\right)^{2}}},
$$

where $\left(x_{t}, y_{t}\right)$ denotes the observed coordinates of the tracked object at time $t$ and $\left(\hat{u}_{t, h}, \hat{v}_{t, h}\right)$ its smoothed representation. Finally, $h$ is a smoothing parameter to be set according to the noise magnitude. Explicit expressions can then be derived for the first- and second-order temporal derivatives of the trajectory positions: respectively, $\hat{\dot{u}}_{t, h}, \hat{\dot{v}}_{t, h}, \hat{\ddot{u}}_{t, h}$ and $\hat{\ddot{v}}_{t, h}$.

\section{B. Smoothing parameter selection}

To have a complete automatic feature extraction method, a way to choose the smoothing parameter $h$ is needed. Hence, for any trajectory $T_{k}$ of size $n_{k}$, an average squared error (ASE) (Härdle et al. [19]) criterion is considered, defined by

$$
A S E(h)=A S E\left(\hat{u}_{t, h}\right)=\frac{1}{n_{k}} \sum_{i=1}^{n_{k}}\left(\hat{u}_{i, h}-u_{i}\right)^{2},
$$

where $u_{i}$ are the "true" values to estimate. A naive approximation $p(h)$ of $A S E(h)$, called the "resubstitution estimate", is done by replacing $u_{i}$ by $x_{i}$ such that :

$$
p(h)=\frac{1}{n_{k}} \sum_{i=1}^{n_{k}}\left(x_{i}-\hat{u}_{i, h}\right)^{2} .
$$

Now, adding and subtracting $u_{t_{i}}$ to (1) leads to

$$
\begin{aligned}
p(h) & =\frac{1}{n_{k}} \sum_{i=1}^{n_{k}}\left(\left(x_{i}-u_{i}\right)+\left(u_{i}-\hat{u}_{i, h}\right)\right)^{2} \\
& =\frac{1}{n_{k}} \sum_{i=1}^{n_{k}} \varepsilon_{i}^{2}+A S E(h)-\frac{2}{n_{k}} \sum_{i=1}^{n_{k}} \varepsilon_{i}\left(\hat{u}_{i, h}-u_{i}\right)(2)
\end{aligned}
$$

with $\varepsilon_{i}=x_{i}-u_{i}$. Considering now a cross validation method,

$$
C V(h)=\frac{1}{n_{k}} \sum_{i=1}^{n_{k}}\left(x_{i}-\hat{u}_{-i, h}\right)^{2}
$$

where $\hat{u}_{-i, h}$ is a "leave-one-out" estimator given by

$$
\hat{u}_{-i, h}=\frac{\sum_{j \neq i} e^{-\left(\frac{i-j}{h}\right)^{2}} x_{j}}{\sum_{j \neq i} e^{-\left(\frac{i-j}{h}\right)^{2}}},
$$

it can be shown that the expectation of the third term of (2) is equal to zero if $\hat{u}_{-i, h}$ is used instead of $\hat{u}_{i, h}$, i.e.,

$$
E\left[-\frac{2}{n_{k}} \sum_{i=1}^{n_{k}} \varepsilon_{i}\left(\hat{u}_{-i, h}-u_{i}\right)\right]=0 .
$$

Moreover, the first term of (2), $\frac{1}{n_{k}} \sum_{i=1}^{n_{k}} \varepsilon_{i}^{2}$, is independent of $h$ so that choosing $h_{\text {opt }}$ such that $C V(h)$ is minimized is equivalent to minimizing, on average, $A S E(h)$. Practically, in order to keep coherence of $h$ as a window regarding the data, candidates $h$ to minimize $C V(h)$ have to be in the same scale than the smallest values of $j-i$ (i.e., $h \geq 1$ ).

\section{Derivation of the trajectory features}

Local orientation of the curve, given by $\gamma_{t}=\arctan \left(\frac{\dot{v}_{t}}{\dot{u}_{t}}\right)$, is first considered. By construction, it is invariant to $2 \mathrm{D}$ translation and scale transformation. To add invariance to 2D rotation, the temporal derivative of $\gamma_{t}$ (i.e., $\dot{\gamma}_{t}$ ) is analyzed. Thus, $\frac{d\left(\tan \gamma_{t}\right)}{d t}=\frac{1}{\cos ^{2} \gamma_{t}} \dot{\gamma}_{t}$. On the other hand:

$$
\frac{d\left(\tan \gamma_{t}\right)}{d t}=\frac{\ddot{v}_{t} \dot{u}_{t}-\ddot{u}_{t} \dot{v}_{t}}{\dot{u}_{t}^{2}}
$$

Then

Also

$$
\dot{\gamma}_{t}=\cos ^{2} \gamma_{t}\left(\frac{\ddot{v}_{t} \dot{u}_{t}-\ddot{u}_{t} \dot{v}_{t}}{\dot{u}_{t}^{2}}\right) \text {. }
$$

Finally,

$$
\cos ^{2} \gamma_{t}=\left(1+\tan ^{2} \gamma_{t}\right)^{-1}=\frac{\dot{u}_{t}^{2}}{\dot{u}_{t}^{2}+\dot{v}_{t}^{2}} .
$$

$$
\dot{\gamma}_{t}=\frac{\ddot{v}_{t} \dot{u}_{t}-\ddot{u}_{t} \dot{v}_{t}}{\dot{u}_{t}^{2}+\dot{v}_{t}^{2}}=\kappa_{t} \cdot\left\|w_{t}\right\|
$$

where $\kappa_{t}=\frac{\ddot{v}_{t} \dot{u}_{t}-\ddot{u}_{t} \dot{v}_{t}}{\left(\dot{u}_{t}^{2}+\dot{v}_{t}^{2}\right)^{\frac{3}{2}}}$ is the local curvature of the trajectory and $\left\|w_{t}\right\|=\left(\dot{u}_{t}^{2}+\dot{v}_{t}^{2}\right)^{\frac{1}{2}}$ the local velocity magnitude at point $\left(u_{t}, v_{t}\right)$. The numerator of $\dot{\gamma}_{t}$ is the determinant of the matrix $\left(\begin{array}{cc}\dot{u}_{t} & \ddot{u}_{t} \\ \dot{v}_{t} & \ddot{v}_{t}\end{array}\right)$ and the denominator $\dot{u}_{t}^{2}+\dot{v}_{t}^{2}=\left\|w_{t}\right\|^{2}$ is the squared velocity magnitude. Thus, $\dot{\gamma}_{t}$ is invariant to rotation (as well as translation and scale invariant since $\gamma_{t}$ is translation and scale invariant). This local feature also captures well both the trajectory shape and the object speed since it is composed of the local curvature and the instantaneous velocity magnitude.

The feature vector representing a trajectory $T_{k}$ extracted in a video shot is then the vector containing the $n_{k}$ successive values of $\dot{\gamma}: V_{k}=\left(\dot{\gamma}_{1}, \dot{\gamma}_{2}, \ldots, \dot{\gamma}_{n_{k}-1}, \dot{\gamma}_{n_{k}}\right)$.

\section{TRAJECTORY MODELING AND SIMILARITY}

In order to efficiently process the invariant trajectory characterization previously described, probabilistic networks, and more specifically HMMs are used since the inherent properties of this modeling help taking into account the temporal evolution of the spatio-temporal information contained in the trajectories. Classical HMMs, relying on GMMs (Gaussian Mixture Models), are designed to model data of sufficient sizes. Hence this modeling hardly treat small trajectories with only few dozens of observations. An original HMM modeling, based on a uniform quantization of the observation space and dealing efficiently with small trajectories, is here proposed.

\section{A. Design of the hidden Markov model}

Hidden Markov models (HMMs) are used to build the needed statistical framework since HMMs inherently express temporal causality. The HMM framework is exploited in a somewhat original way since the HMMs states are given by properly quantized values of $\dot{\gamma}$. To determine the HMMs state values, the distribution of $\dot{\gamma}$ of the considered trajectories is studied. For each trajectory $T_{k}$, an interval $\left[B_{1, k}, B_{2, k}\right]$ is defined, centered around the mean value $m_{k}$ of $\dot{\gamma}$ and containing a given percentage $P_{v}$ of the computed $\dot{\gamma}$. Experimentally, using $P_{v}$ equal to $95 \%$ gave the best efficiency so that in the following, $P_{v}$ will be fixed at a $95 \%$ value. Then, a quantization is performed on $\left[B_{1, k}, B_{2, k}\right]$ into a number $N_{k}^{\prime}$ of bins, named the interior states. This is illustrated in Fig. 1 which presents the quantization performed on the considered $P_{v}$ percentage (i.e., in $\left[B_{1, k}, B_{2, k}\right]$ ) of $\dot{\gamma}$ observations. Two boarding states (unlimited states) are also defined by ]$\left.-\infty, B_{1, k}\right]$ and $\left[B_{2, k},+\infty[\right.$, so that the total number of states corresponding to a trajectory $T_{k}$ is $N_{k}=N_{k}^{\prime}+2$ where states 

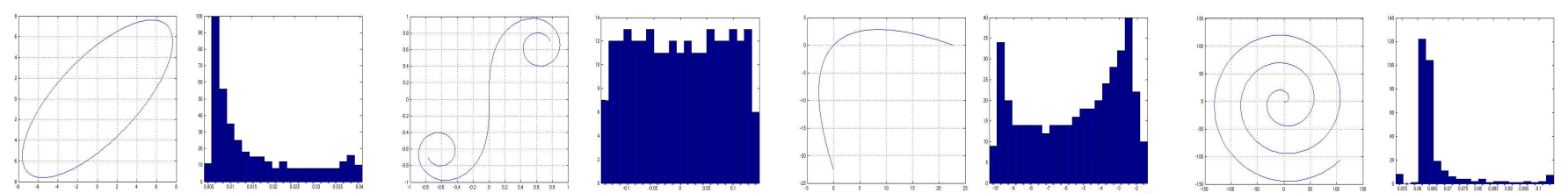

Fig. 2. Samples of synthetic trajectories (an ellipse, a clothoid, a parabola and a spiral) and their associated $\dot{\gamma}$ histograms in the intervals $\left[B_{1, k}, B_{2, k}\right]\left(P_{v}=95 \%\right.$ and $\left.N=21\right)$

$S_{1}$ and $S_{N_{k}}$ are defined by $\left.]-\infty, B_{1, k}\right]$ and $\left[B_{2, k},+\infty[\right.$. Fig. 2 presents four synthetic trajectories and their corresponding histograms in the considered quantization. This quantizationbased HMM framework is now denoted as QHMM.

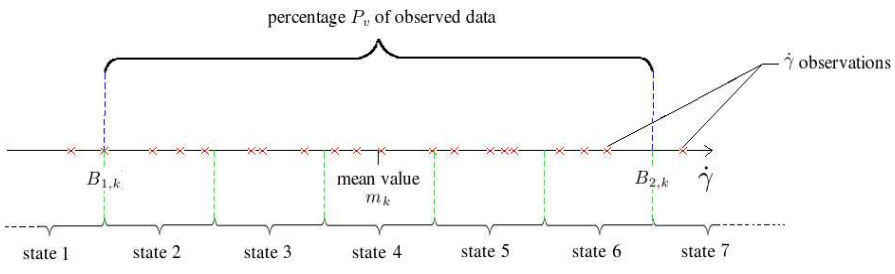

Fig. 1. Quantization performed on the $\dot{\gamma}$ data corresponding to a trajectory $T_{k}$ by using five bins, corresponding to five interior states $\left(N_{k}^{\prime}=5\right.$ and, thus, $N_{k}=7$ ).

Methods that use QHMM to treat video trajectories have already been designed in [20] (that will be denoted by "global QHMM"). The overall idea is here somewhat different since a single and characterizing interval per trajectory is now considered, whereas only one global interval (on all the $\dot{\gamma}$ data from all the considered trajectories) was used before, considering the two boarding states as outlier measurements. This new proposed technique allows to consider the extreme data values of a trajectory as important information corresponding to significant phases of the movement of a precise mobile.

The QHMM which models the trajectory $T_{k}$ is now characterized by:

- the state transition matrix $A=\left\{a_{i j}\right\}$ with

$$
a_{i j}=P\left[q_{t+1}=S_{j} \mid q_{t}=S_{i}\right], \quad 1 \leq i, j \leq N_{k},
$$

where $q_{t}$ is the state variable at instant $t$ and $S_{i}$ is its value (i.e., the $i$ th bin of the quantized histogram);

- the initial state distribution $\pi=\left\{\pi_{i}\right\}$, with $\pi_{i}=P\left[q_{1}=\right.$ $\left.S_{i}\right], \quad 1 \leq i \leq N_{k}$

- the conditional observation probabilities $B=$ $\left\{b_{i}\left(\dot{\gamma}_{t}\right)\right\}, \quad 1 \leq i \leq N_{k}$, where $b_{i}\left(\dot{\gamma}_{t}\right)=P\left[\dot{\gamma}_{t} \mid q_{t}=S_{i}\right]$, since the computed $\dot{\gamma}_{t}$ are the observed values.

The conditional observation probabilities $P\left[\dot{\gamma}_{t} \mid q_{t}=S_{i}\right]$ are defined, in $\left[B_{1, k}, B_{2, k}\right]$, as Gaussian distributions of means $\mu_{i}$ (i.e., the median value of the histogram bin $S_{i}$ ). Their standard deviations $\sigma$ do not depend on the state and are specified so that the intervals $\left[\mu_{i}-\sigma, \mu_{i}+\sigma\right]$ correspond to the bin widths. These conditional observations probabilities are then normalized such that, for any observation $\dot{\gamma}$, $\sum_{i=1 \ldots N} P\left[\dot{\gamma}_{t} \mid q_{t}=S_{i}\right]=1$. Outside of $\left[B_{1, k}, B_{2, k}\right]$, the observation are considered to belong to the corresponding boarding state. An illustration of the conditional observation probabilities is presented in Fig. 3.

This conditional observation model has one very important advantage. Considering that an observation may (even with very small probabilities) belong to any other state, this

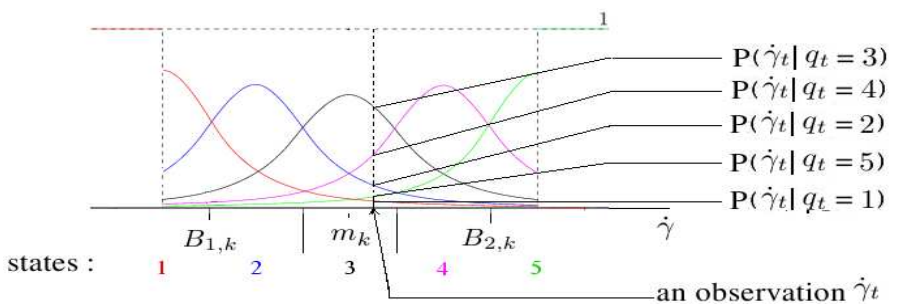

Fig. 3. Modeling of the conditional observation probabilities for a trajectory $T_{k}$, using a number of states $N_{k}=5$.

method helps dealing with very small trajectories, preventing from having zero values when estimating matrix $A$ in the training stage by lack of measure (especially in case of short trajectories). Otherwise, if matrix $A$ have zero values, infinite distances will be found between two (possibly "close") trajectories.

To estimate $A$ and $\pi$ in this QHMM modeling, a leastsquare error technique used in [15] is adapted here, where the QHMMs are assimilated to a count process. If $H_{t}^{(i)}=$ $P\left(\dot{\gamma}_{t} \mid q_{t}=i\right)$ (corresponding to a weight for the count process), empirical estimates of $A$ and $\pi$ (for a trajectory $k$ of size $n_{k}$ ) are given by, for $1 \leq i \leq N_{k}$ and $1 \leq j \leq N_{k}$ :

$$
a_{i j}=\frac{\sum_{t=1}^{n_{k}-1} H_{t}^{(i)} H_{t+1}^{(j)}}{\sum_{t=1}^{n_{k}-1} H_{t}^{(i)}} \text { and } \pi_{i}=\frac{\sum_{t=1}^{n_{k}} H_{t}^{i}}{n_{k}} .
$$

As illustration, examples of a real trajectories are showed in Fig. 4, their smoothed counterparts as well as the estimated values of $A$ and $\pi$ coefficients for their associated QHMMs.

In contrast, in the HMM framework relying on Gaussian mixture models (GMMs) introduced in [43] to model trajectories and their temporal evolution, the number of states remains difficult to set (it relies on a validity score that requires a balancing factor to be fixed), whereas in the next section, a statistical automatic QHMM state number selection is proposed. Furthermore, to have an efficient approximation of the GMMs, the trajectory size should be much larger than the number of Gaussian mixture components (used for the conditional observation distribution) times the number of states [43], whereas the novel QHMM method is developed to handle trajectories of any sizes.

\section{B. Choosing the number of HMM states}

In the QHMM framework described above, the number of interior states $N_{k}^{\prime}$ (in $\left[B_{1, k}, B_{2, k}\right]$ ) used to define a model describing a trajectory $T_{k}$ remains undefined. To efficiently compute this parameter, a statistical decision based upon a balance between the number of states and the confidence in the histogram values is developed. The aim is to define a criterion such that both the number of states (to prevent from 
overfitting) and the size of the corresponding state histogram values confidence intervals (to have a reliable histogram representation) would be as small as possible.

Considering $\Theta_{j}$ the "real" value corresponding to bin $j$ (i.e., state $j$ ) of the normalized histogram representing the distribution of the $\dot{\gamma}$ of a trajectory $T_{k}$ (of size $n_{k}$ ), and its estimator $\hat{\Theta}_{j}$ defined as the proportion of observed $\dot{\gamma}$ in the considered bin by

$$
\begin{aligned}
& \text { where } \\
& K_{i}=\sum_{l=1}^{n_{k}^{\prime}} X_{i, l}=\sum_{l=1}^{n_{k}^{\prime}} \mathbb{1}_{\left\{\dot{\gamma}_{l} \in S_{i}\right\}}, i=2 \ldots N_{k}^{\prime}+1
\end{aligned}
$$$$
\hat{\Theta}_{j}=\frac{K_{j}}{n_{k}^{\prime}},
$$

is the number of observations in the bin $S_{i}$, and

$$
n_{k}^{\prime}=\sum_{i=2}^{N_{k}^{\prime}+1} K_{i}
$$

is the number of observations in $\left[B_{1, k}, B_{2, k}\right]$.

$X_{i, l}=\mathbb{1}_{\left\{\dot{\gamma}_{l} \in S_{i}\right\}}$ is the indicator function of belonging of $\dot{\gamma}_{l}$ in the interior states, and the hypothesis that $X_{i, l}$ follows a Bernoulli law on $\left[B_{1, k}, B_{2, k}\right]$ is done.

Then, using the central limit theorem [14],

$$
\frac{\hat{\Theta}_{j}-\mathbb{E}\left[\hat{\Theta}_{j}\right]}{\sqrt{\mathbb{V}\left[\hat{\Theta}_{j}\right]}} \stackrel{\mathcal{L}}{\rightarrow} \mathcal{N}(0,1), \forall j=2 \ldots N_{k}^{\prime}+1 .
$$

So, asymptotically,

$$
\hat{\Theta}_{j} \rightarrow \mathcal{N}\left(\mathbb{E}\left[\hat{\Theta}_{j}\right], \mathbb{V}\left[\hat{\Theta}_{j}\right]\right), \forall j=2 \ldots N_{k}^{\prime}+1 .
$$

$\hat{\Theta}_{j}$ is (trivially) an unbiased estimator of $\Theta_{j}$, so that the confidence interval $I C_{95}$ (with a confidence percentage of 95\%) of $\Theta_{j}$ can be defined by

$$
I C_{95}\left(\Theta_{j}\right)=\left[\hat{\Theta}_{j}-\alpha_{95} \mathbb{V}\left[\hat{\Theta}_{j}\right], \hat{\Theta}_{j}+\alpha_{95} \mathbb{V}\left[\hat{\Theta}_{j}\right]\right],
$$

where the $\alpha_{95}$ quantile is the value ensuring that, considering eq. 3,

$$
P\left(\Theta_{j} \in\right] \hat{\Theta}_{j}-\alpha_{95} \mathbb{V}\left[\hat{\Theta}_{j}\right], \hat{\Theta}_{j}+\alpha_{95} \mathbb{V}\left[\hat{\Theta}_{j}\right][) \geq 0.95 .
$$

The random variable $X_{j, l}$ follows a Bernoulli law so that

$$
\mathbb{V}\left[X_{j, l}\right]=\Theta_{j}\left(1-\Theta_{j}\right)
$$

Using $\hat{\Theta}_{j}$ as an unbiased estimator of $\Theta_{j}, \mathbb{V}\left[X_{j, l}\right]$ can be approximated by

$$
\mathbb{V}\left[X_{j, l}\right] \simeq \hat{\Theta}_{j}\left(1-\hat{\Theta}_{j}\right)
$$

Hence,

$$
\begin{aligned}
\mathbb{V}\left[\hat{\Theta}_{i}\right] & =\mathbb{V}\left[\frac{1}{n_{k}^{\prime}} \sum_{l=1}^{n_{k}^{\prime}} X_{i, l}\right]=\frac{1}{n_{k}^{\prime 2}} \mathbb{V}\left[\sum_{l=1}^{n_{k}^{\prime}} X_{i, l}\right] \\
& =\frac{1}{n_{k}^{\prime 2}} n_{k}^{\prime} \mathbb{V}\left[X_{i, l}\right] \simeq \frac{\hat{\Theta}_{i}\left(1-\hat{\Theta}_{i}\right)}{n_{k}^{\prime}}
\end{aligned}
$$

The confidence interval $I C_{95}\left(\Theta_{j}\right)$ has a size $\left|I C_{95}\left(\Theta_{j}\right)\right|$ which can be estimated by

$$
\begin{aligned}
\left|I C_{95}\left(\Theta_{j}\right)\right| & =2 \alpha_{95} \mathbb{V}\left[\hat{\Theta}_{j}\right] \simeq 2 \alpha_{95} \frac{\hat{\Theta}_{j}\left(1-\hat{\Theta}_{j}\right)}{n_{k}^{\prime}} \\
& \simeq 2 \alpha_{95} \frac{K_{j} n_{k}^{\prime}-K_{j}}{n_{k}^{\prime 3}} \simeq 2 \alpha_{95} \frac{K_{j}\left(n_{k}^{\prime}-1\right)}{n_{k}^{\prime 3}} .
\end{aligned}
$$

Considering now the mean value $m_{I C, k}$ of $\left|I C_{95}\left(\Theta_{j}\right)\right|$ for the trajectory $T_{k}$,

$$
m_{I C, k} \simeq \frac{\sum_{j=2 \ldots N_{k}^{\prime}+1}\left|I C_{95}\left(\Theta_{j}\right)\right|}{N_{k}^{\prime}} .
$$

$\left|I C_{95}\left(\Theta_{j}\right)\right|$ (and so $\left.m_{I C, k}\right)$ is a decreasing function of $N_{k}^{\prime}$ since $K_{j}$ is a decreasing function of $N_{k}^{\prime}$. The decision criterion based upon a balance between the number of states $N_{k}^{\prime}$ and the mean size of the confidence interval $m_{I C, k}$ is then defined by choosing $\tilde{N}_{k}^{\prime}$ minimizing $m_{I C, k}+\delta N_{k}^{\prime}$, such that

$$
\tilde{N}_{k}^{\prime}=\arg \min _{N_{k}^{\prime}}\left(m_{I C, k}+\delta N_{k}^{\prime}\right) \text {. }
$$

Choosing $\delta$, which is a scaling parameter aiming at helping the comparison of $m_{I C, k}$ and $N_{k}^{\prime}$ by ensuring these two values to evolve in the same scale, is still needed. Considering a given distribution, the asymptotic estimations of the proportions $\hat{\Theta}_{j}, j=2 \ldots N_{k}^{\prime}+1$ are constant. Hence, $m_{I C, k}$ is a decreasing function of $n_{k}^{\prime}$, and, thus, it can be assumed that $\delta$ is also a decreasing function of $n_{k}^{\prime}$. In order to determine the function $\delta\left(n_{k}^{\prime}\right)$, a decreasing function $\delta\left(n_{k}^{\prime}\right)=\frac{\beta}{n_{k}^{\prime}}$ (decreasing function having the same form that $\left.m_{I C, k}\right)$ is empirically considered.

First, the choice of a $\hat{\delta}$ constant is done by considering a value which gives somewhat compact distributions of $\tilde{N}_{k}^{\prime}$ for the considered classes of similar trajectories (see Fig. 8 for the classes of trajectories), by maximizing the inter-class distances while minimizing the intra-class distances (first order linear discriminant analysis on $\delta$ ). Then, choosing a representative value of $\tilde{N}_{C_{i}}^{\prime}$ for the trajectories of each class $C_{i}$ (for example the mean of the $\tilde{N}_{k}^{\prime}$ found using $\hat{\delta}$ for the instances of a class, leaving out the isolated points), the $\delta$ intervals that leads to this representative $\tilde{N}_{C_{i}}^{\prime}$ value (for a class $C_{i}$ ) versus the sizes of the trajectories are then considered.

As a result, a regression was processed on the upper and lower bound values of these intervals, considering that

$$
\delta\left(n_{k}^{\prime}\right)=\frac{\beta}{n_{k}^{\prime}}+e_{k} .
$$

Using a least square error estimation scheme, the value $\beta$ minimizing $\sum_{i} e_{k}^{2}$ is found. Fig. 5 shows that a regression using an inverse function of $n_{k}^{\prime}$ with a least square error estimation gives very satisfying results, validating the hypothesis that $\delta$ is a decreasing function of $n_{k}^{\prime}$ and more specifically an inverse function of $n_{k}^{\prime}$. This first determined function $\delta\left(n_{k}^{\prime}\right)$ is then used to choose a new number of states $\tilde{N}_{k}^{\prime}$ for the whole set of considered trajectories, leading to a new regression and so on until the function $\delta\left(n_{k}^{\prime}\right)$ was stable (no more changes in the states sequence associated to the set of trajectories). This method enables to have a relevant function $\delta\left(n_{k}^{\prime}\right)$ for any class of trajectory. In the experiments, it has been found that $\beta=0.0175$, so that $\delta\left(n_{k}^{\prime}\right)=\frac{0.0175}{n_{k}^{\prime}}$ is an efficient scaling coefficient allowing automatic states number choose, for any trajectory of any class.

Then, to process a relevant comparison (i.e comparing HMMs having the same number of states), an unique number $\tilde{N}^{\prime}$ is needed for the whole set of trajectories. Using the function $\delta\left(n_{k}^{\prime}\right)$ described above, the state number $\tilde{N}^{\prime}$ to consider in the intervals $\left[B_{1, k}, B_{2, k}\right]$ is defined by

$$
\tilde{N}^{\prime}=\arg \min _{N^{\prime}} \sum_{k}\left(m_{I C, k}+\delta N^{\prime}\right),
$$

when considering the data corresponding to the whole set of trajectories (Fig. 6). 

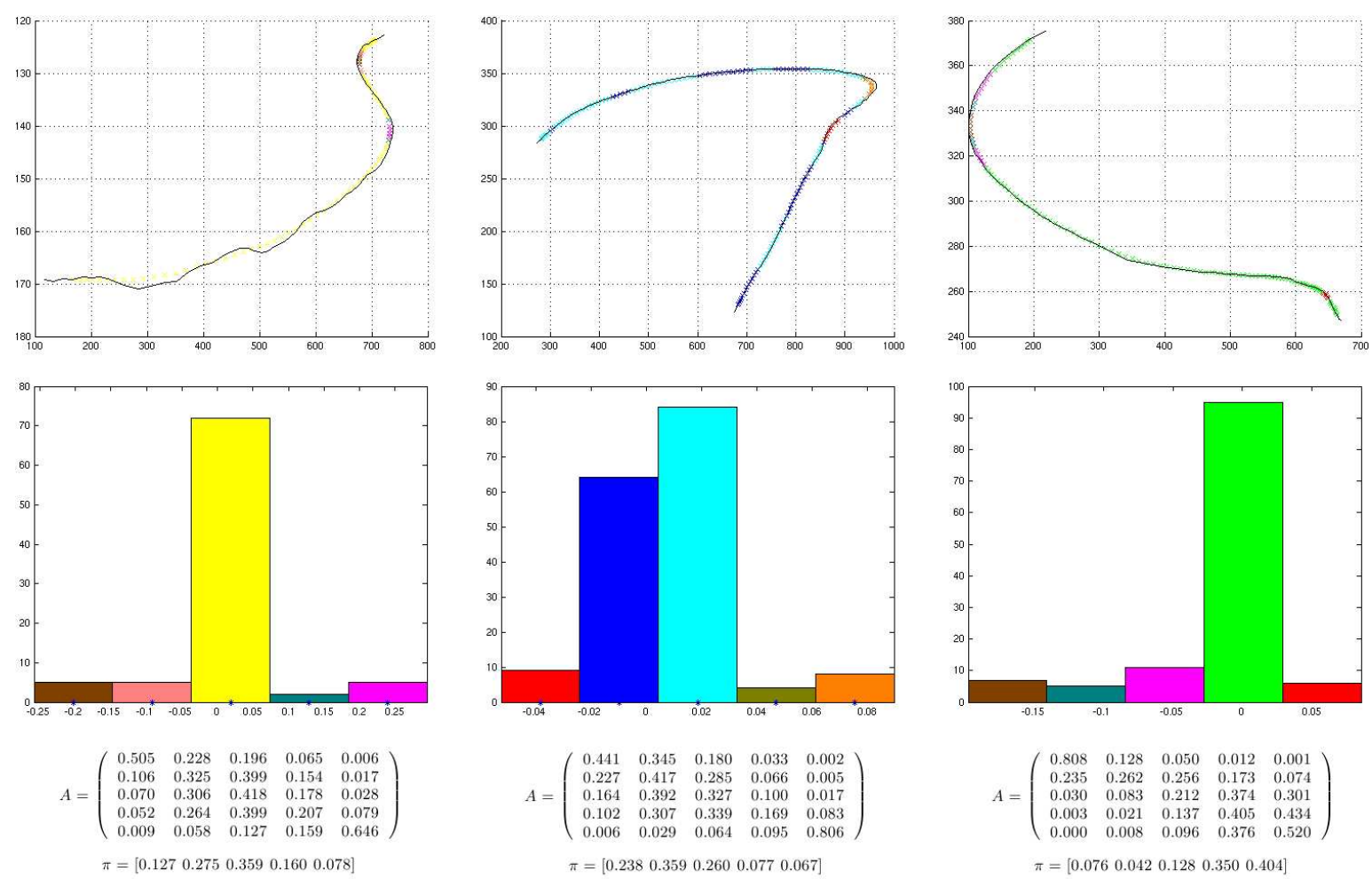

Fig. 4. Upper part: Plots of real trajectories (extracted from Formula One race video shots) and their smoothed counterparts. Colors of the curve points stand for the different state values and correspond to the histogram bin colors (colors have been chosen randomly since the states are different for each trajectory). Middle part: histograms of the three trajectories state values. Lower part: corresponding estimated transition matrices $A$ and initial state distributions $\pi$.

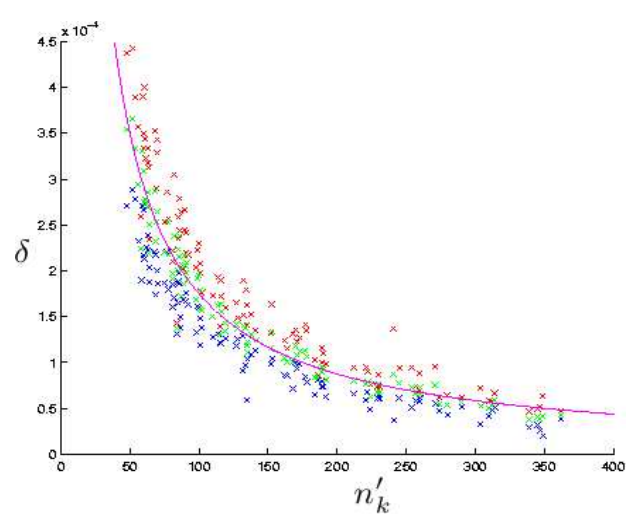

Fig. 5. $\delta$ intervals leading to a chosen "good" $N^{\prime}$ according to data sizes (trajectory or group of trajectories from different classes). The red and blue points respectively correspond to the upper and lower bound, the green ones corresponding to the means of the intervals. The purple function is the regression obtained on the red and blue points, using a least square error estimator and an inverse function of the data sizes.

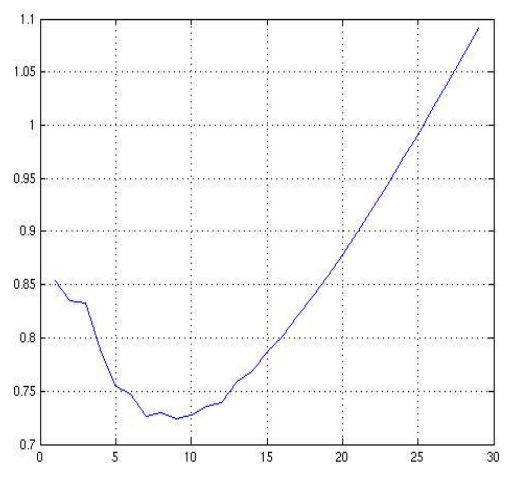

Fig. 6. function representing the balance $\sum_{k}\left(m_{I C, k}+\delta N^{\prime}\right)$ used to choose the number of interior states $N^{\prime}$ of the QHMM models when considering the whole set of data corresponding to the 8 classes of Formula One trajectories (Fig. 8).

\section{Similarity measure}

To compare two trajectories, a similarity measure has to be defined. To this end, the built QHMM framework is exploited. To compare trajectories, a cross distance based on the distance $D$ between HMMs proposed by Rabiner [44] is defined. Given two HMMs represented by their parameter sets $\lambda_{i}$ and $\lambda_{j}$ $\left(\lambda_{i}=\left(A_{i}, B_{i}, \pi_{i}\right)\right)$, the distance $D$ is defined by

$$
D\left(\lambda_{i}, \lambda_{j}\right)=\frac{1}{T}\left[\log P\left(O^{(j)} \mid \lambda_{j}\right)-\log P\left(O^{(j)} \mid \lambda_{i}\right)\right]
$$

where $O^{(j)}=\left\{\dot{\gamma}_{1}, \dot{\gamma}_{2}, \ldots, \dot{\gamma}_{n_{j}}\right\}$ is the sequence of measures used to train the model $\lambda_{j}$ and $P\left(O^{(j)} \mid \lambda_{i}\right)$ expresses the probability of observing $O^{(j)}$ with model $\lambda_{i}$ (computed using a Viterbi algorithm). To be used as a similarity measure, a symmetrized version is required:

$$
D_{s}\left(\lambda_{i}, \lambda_{j}\right)=\frac{1}{2}\left[D\left(\lambda_{i}, \lambda_{j}\right)+D\left(\lambda_{j}, \lambda_{i}\right)\right] .
$$

In the presented method, a specific modeling is associated to each trajectory $T_{k}$, and is defined by the interval $\left[B_{1, k}, B_{2, k}\right]$. Hence, to compare two trajectories $T_{i}$ and $T_{j}$, the parameter sets $\lambda_{i}^{j}, \lambda_{i}^{i}, \lambda_{j}^{j}$ and $\lambda_{j}^{i}$ are computed, where $\lambda_{i}^{j}$ correspond to the parameters found for the trajectory $T_{j}$ when considering the model associated to the trajectory $T_{i}$ ( $\dot{\gamma}$ interval). Then, the cross symmetrized distance $D_{c}$ between two trajectories $T_{i}$ and $T_{j}$ is then defined by :

$D_{c}\left(\lambda_{i}, \lambda_{j}\right)=\frac{1}{4}\left[D\left(\lambda_{i}^{i}, \lambda_{j}^{i}\right)+D\left(\lambda_{j}^{i}, \lambda_{i}^{i}\right)+D\left(\lambda_{i}^{j}, \lambda_{j}^{j}\right)+D\left(\lambda_{j}^{j}, \lambda_{i}^{j}\right)\right]$. 


\section{VIDEO UNDERSTANDING TASKS}

\section{A. Recognition of learned classes of dynamic video content}

The problem of recognizing events, or equivalently, of retrieving instances of known classes of events in videos is here considered. Each class is first modeled by a set of $\mathrm{QH}-$ MMs corresponding to representative trajectories (those used in the training step, i.e., the initial members of the classes). Recognition is then performed by assigning the processed trajectory to the nearest class. The distance to a class is defined using the average link method and $D_{c}$ such that:

$$
D_{a l}\left(T_{k}, C_{i}\right)=\frac{\sum_{T_{l} \in C_{i}} D_{c}\left(T_{k}, T_{l}\right)}{\# C_{i}} .
$$

\section{B. Detection of unexpected events}

Detecting unexpected (or equivalently, rare or abnormal) events is of interest in many applications. This issue has been tackled using the same QHMM-based framework. First, a set of predefined (or learned) classes is considered, once again represented by the estimated QHMMs of the class initial members. For each class $C_{i}$, the most representative trajectory $T_{l_{i}}$ (i.e, the trajectory with the smallest mean distance to the other trajectories of $C_{i}$ ) is found, and the distribution of the intra-class distances to $T_{l_{i}}$ is computed (with the other trajectories of $C_{i}$ ). For each class $C_{i}$, the maximum of these intra-class distance values is denoted by $R_{i}$, and the standard deviation of these intra-class distance values by $\sigma_{i}$. A test trajectory $T_{k}$ is then defined to be an unexpected event if, for every class $C_{i}, D_{c}\left(T_{k}, T_{l_{i}}\right)>R_{i}+\sigma_{i}$, which endures a low false alarm rate.

\section{OTHER METHODS FOR COMPARISON PURPOSE}

To our knowledge, no previous works of video trajectory analysis considered the overall view adopted in this paper. By considering a feature having those invariances and characteristics (considering both the trajectory shapes and speed evolutions), this work considers the trajectory as a dynamical pattern while the other ones consider the trajectories as attached to the camera point of view. Therefore, in order to compare in a relevant way with other methods, new trajectory comparison techniques were developed to put forward the properties (spatio-temporal consideration and efficient processing of small trajectories) of the developed QHMM method.

The methods presented for the recognition of dynamic video content task and for the QHMM state number selections stands similarly when considering the next three presented distances to be compared with the QHMM method.

\section{A. Global QHMM distance}

A method based on QHMM for trajectory analysis (classification, clustering and rare event detection) [20] [21] was already developed. This later will be denoted now by global QHMM since the QHMM were used to model the whole $\dot{\gamma}$ distribution (for the entire set of data associated to all the considered trajectories) and not dedicated to the precise and specific modeling of each trajectories. This global QHMM method will be used to compare with the new one.

\section{B. Bhattacharyya distance between histograms}

Based on the global QHMM trajectory analysis method, and in order to assess the importance of introducing temporal causality, i.e., transitions between states, a Bhattacharyya distance-based classification method has been implemented. The Bhattacharyya distance $D_{b}$ between two (normalized) histograms $h_{i}$ and $h_{j}$ of features $\dot{\gamma}_{t}$, respectively corresponding to two trajectories $T_{i}$ and $T_{j}$, is defined by

$$
D_{b}\left(T_{i}, T_{j}\right)=\sqrt{1-\sqrt{\sum_{q=1}^{N} h_{q}^{i} h_{q}^{j}}}
$$

where $h_{q}^{i}$ is the histogram value of bin $q$ for trajectory $T_{i}$. Similarly to the global QHMM method, the test trajectory $T_{k}$ is assigned to the nearest class using an average link method (see eq. 5 , but using here $D_{b}$ ).

\section{Crossed Bhattacharyya distance between histograms}

To highlight the relevance of considering temporal causality in the novel QHMM method, a cross Bhattacharyya distancebased classification method has been implemented. The standard Bhattacharyya distance between histograms was enlarged to the crossed Bhattacharyya distance $D_{c b}$ between two trajectories $T_{i}$ and $T_{j}$ defined by

$$
D_{c b}\left(T_{i}, T_{j}\right)=\sqrt{1-\sqrt{\sum_{q_{i}=1}^{N_{i}} h_{q_{i}}^{i} h_{q_{i}}^{j}}}+\sqrt{1-\sqrt{\sum_{q_{j}=1}^{N_{j}} h_{q_{j}}^{i} h_{q_{j}}^{j}}}
$$

where $h_{q_{j}}^{i}$ is the histogram value of bin $q_{j}$ (corresponding to the bin $q$ of the QHMM modeling associated to trajectory $T_{j}$ ) when considering the trajectory $T_{i}$.

\section{D. $H M M / G M M$ modeling}

Inspired by the work of Porikli, the proposed HMM/GMM distance [43] was extended to the analysis of the $\dot{\gamma}_{t}$ feature. This comparison enables to highlight some advantages of the QHMM method in term of results (QHMM may deal with small trajectories, not subject to overfitting when data distributions are difficult to model using Gaussian mixtures). An ergodic HMM was computed where the modeling of observation $\left(\dot{\gamma}_{t}\right.$ values) probabilities were done using Gaussian Mixture Models. Initialization was done using a k-means algorithm. To determine the number of states of this HMM modeling, a Bayesian Information Criterion (BIC) has been used. Using this HMM/GMM framework to classify trajectories, one HMM/GMM will be created for each trajectory, showing the relevance of the QHMM when dealing with small data sizes. The different considered tasks where realized using the same methods that with the QHMM method and the standard Rabiner distance $D_{s}$ (see eq. 4).

\section{E. SVM classification method}

To compare with an usual and efficient classification tool, a Support Vector Machine (SVM) trajectory classification method [7] was computed. The SVM method proposed in [20] has been considered, where a trajectory is represented by its global QHMM parameters (since every trajectory is modeled using the same quantization scheme). Hence, for each 
trajectory $T_{k}$, a vector $X_{k}$ containing the HMM parameters $\lambda_{k}$ of the trajectory is created. A SVM classification technique with a Gaussian RBF (radial basis function) kernel was used. The reported results were obtained using the "one against all" classification scheme. For this classification method, the number of state $N$ parameter selection method of subsection IV-B was also processed.

\section{EXPERIMENTS}

\section{A. Video trajectories}

Real trajectories have been extracted from Formula One (Fig. 7) and Alpine skiing TV programs (downhill and slalom races, Fig. 9) filmed with several cameras. Trajectories are computed with a tracking method using a color-based particle filter [40]. Background motion due to camera panning, tilting and zooming is estimated and compensated in the tracking procedure [18]. Trajectory shapes supplied by this method are thus fairly similar to the real 3D trajectories of Formula Ones and skiers (up to an homography, since the 3D motion is planar, examples are plotted on Fig. 8 and 10).

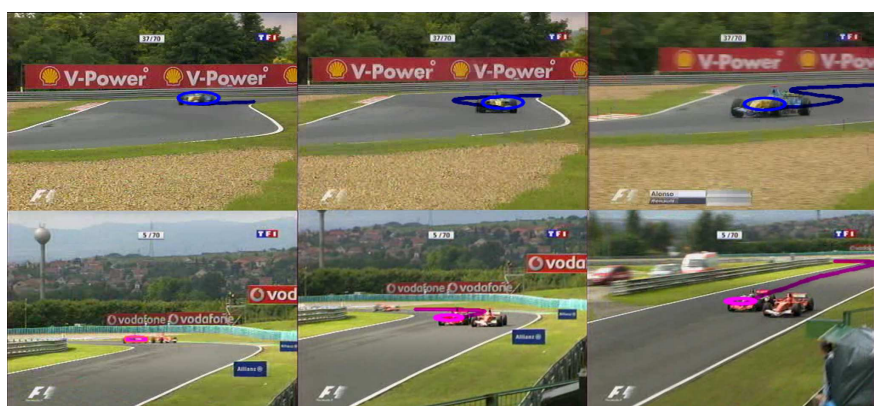

Fig. 7. Images from video shots acquired by two different cameras in Formula One TV program at two different places on the circuit. The computed trajectories are overprinted on the images.

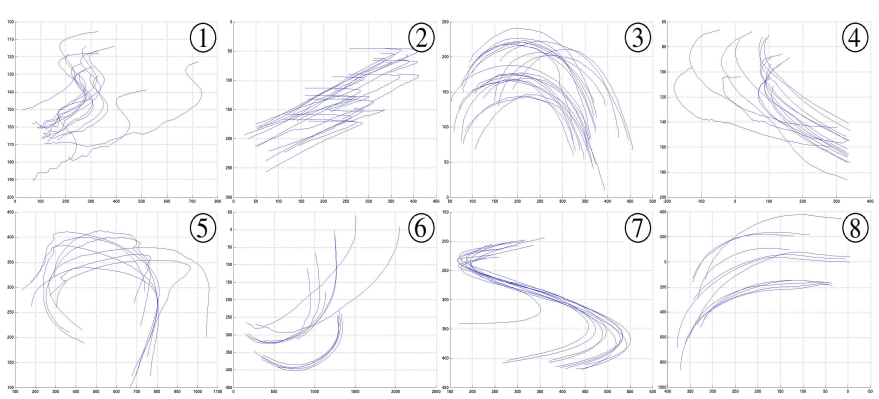

Fig. 8. Plots of the 8 classes of trajectories (125 trajectories) of a Formula One race video, each box contains a different class. A class of trajectories is composed of trajectories extracted from shots acquired by the same camera. The different classes correspond to different cameras placed throughout the circuit at strategic turns.

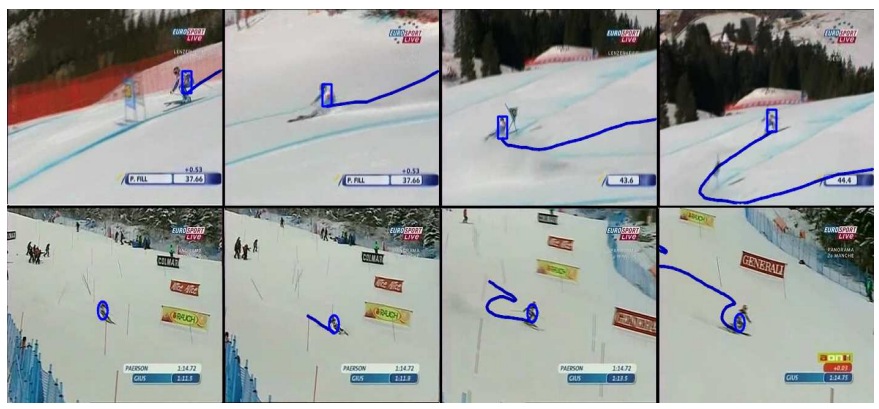

Fig. 9. Images from ski video shots from two different races (the first one from a downhill race and the other one from a slalom race). The computed trajectories are overprinted on the images.

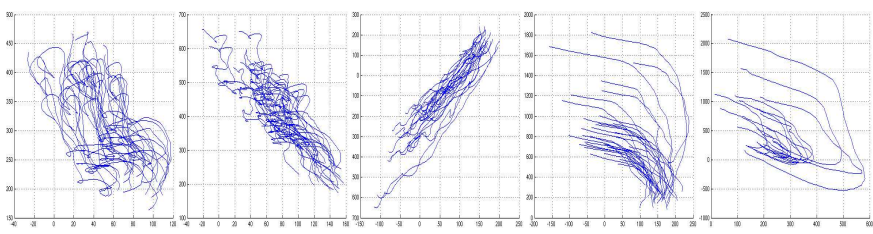

Fig. 10. Plots of the 5 classes of skier trajectories (134 trajectories), each box contains a different class. A class of trajectories is composed of trajectories extracted from shots acquired by the same camera. The three classes on the left correspond to slalom trajectories and the two ones on the right correspond to downhill trajectories.

\section{B. Results on supervised recognition}

Results regarding the recognition task are now reported. QHMM-based method has been compared with the histogram comparison technique based on the cross Bhattacharyya distance, the HMM/GMM modeling, the global QHMM method, the Bhattacharyya distance and the SVM method outlined in Section VI. To evaluate the performances, a leave-one-out cross validation [22] was adopted. Table II and Table III contain respectively the classification results for the set of Formula One and for the set of ski video trajectories (corresponding classes are presented in Fig. 8 and Fig. 10).

This evaluation on real videos gave very satisfying results since accurate classifications were performed with the QHMM method, while the other techniques gave less accurate results. The comparison with the method based on the cross Bhattacharyya distance, which also supplied interesting results, shows the importance of the temporal causality modeled by the QHMMs. The relevance of considering each trajectory individually is highlighted by the comparison with the global QHMM method and the Bhattacharyya distance based technique. These two latest methods (and the SVM one) yielded less accurate results, and the classification results were better when considering 6 classes than 4 classes, showing the lack of stability of these methods due to the global quantization modeling. HMM/GMM modeling fails to classify trajectories as efficiently as the other proposed methods (highlighting the flexibility of the QHMM modeling versus HMM/GMM modeling when considering small sets of data).

A major advantage of considering invariant features is to be able to handle incomplete background motion elimination. Indeed, the camera motion estimation step may be inaccurate, which generates residual effects o, the computed trajectories as shown in Figs. 8 and 10. All the classes present translation errors, and several contains important scale errors (e.g., classes 6 and 7 of Fig. 8, two classes on the right of Fig. 10).

Correct classification results were also obtained when considering the "slalom" and "downhill" classes of ski trajectories (the "slalom" class composed with the three classes on the left of Fig. 10 and the class "downhill" composed with the two classes on the right of Fig. 10) with each tested method. Classifications of "tight turn" (class 2 in Fig. 8), "light turn" (class 4 and 8 in Fig. 8), "chicane" (class 1 and 7 in Fig. 8) and "U-turn" (class 3, 5 and 6 in Fig. 8) Formula One trajectories also gave correct results.

These last results highlight the relevance of the chosen feature $\dot{\gamma}$ for analyzing the dynamic content of video motions acquired by several different cameras. 


\begin{tabular}{|c|c|c|c|c|c|c|c|c|c|}
\hline & Class 1 & Class 2 & Class 3 & Class 4 & Class 5 & Class 6 & Class 7 & Class 8 & Status \\
\hline$R_{i}+\sigma_{i}$ & 0.0013 & 0.0141 & 0.1189 & 0.0069 & 0.0055 & 0.2077 & 0.0290 & 0.1167 & \\
\hline Accident 1 & 0.0031 & 0.1823 & 0.3056 & 0.0467 & 0.0303 & 0.4667 & 0.1285 & 0.2313 & detected \\
\hline Track off & 0.0199 & 0.3487 & 0.3388 & 0.1260 & 0.0064 & 0.3918 & 0.0883 & 0.1544 & detected \\
\hline Accident 2 & 0.0070 & 0.1000 & 0.1788 & 0.1260 & 0.0083 & 0.2885 & 0.0373 & 0.1381 & detected \\
\hline Safety car & 0.0117 & 0.1069 & 0.1385 & 0.0942 & 0.0353 & 0.2673 & 0.05367 & 0.1362 & detected \\
\hline
\end{tabular}

TABLE I

DETECTION THRESHOLdS ARE SUPPLIED IN THE SECOND ROW FOR THE (LEARNED) ClASSES $C_{i}$ USED IN THE DETECTION TASK OF UNEXPECTED EVENTS. THE FOLLOWING ROWS CONTAIN THE DISTANCES BETWEEN THE UNEXPECTED EVENTS TRAJECTORIES AND THE 8 REGULAR CLASSES (PLOTTED IN FIG. 8 ). THE EVENTS 'ACCIDENT 1 ', 'TRACK OFF' AND 'SAFETY CAR' WERE SHOT BY THE CAMERA CORRESPONDING TO CLASS 1, WHEREAS 'ACCIDENT 2' CORRESPONDS TO CLASS 2.

\begin{tabular}{|c|ccc|}
\hline & Percentage & of & correct \\
\hline & 4 & 6 & 8 \\
\hline \# classes & 100 & 99 & 96 \\
\hline QHMM & 98.2 & 98 & 95.2 \\
\hline Crossed-Bhattacharyya & 96.4 & 99 & 94.4 \\
\hline Global QHMM & 94.5 & 96 & 93.6 \\
\hline Bhattacharyya & 96.4 & 99 & 92.8 \\
\hline SVM & 98.2 & 96 & 80 \\
\hline HMM/GMM & & &
\end{tabular}

TABLE II

COMPARISON OF THE RECOGNITION PERCENTAGES FOR THE TRAJECTORIES EXTRACTED FROM FORMULA ONE VIDEOS, USING THE LEAVE-ONE-OUT CROSS VALIDATION TECHNIQUE. THE CONSIDERED GROUPS OF 4 AND 6 CLASSES ARE RESPECTIVELY COMPOSED WITH THE CLASSES 1 TO 4 AND 1 TO 6 IN FIG. 8.

\begin{tabular}{|c|c|}
\hline & Percentage of correct classification \\
\hline QHMM & 92.4 \\
\hline Crossed-Bhattacharyya & 91.7 \\
\hline Global QHMM & 91.7 \\
\hline Bhattacharyya & 91.7 \\
\hline SVM & 91 \\
\hline HMM/GMM & 78.2 \\
\hline
\end{tabular}

COMPARISON OF THE RECOGNITION PERCENTAGES FOR THE 5 CLASSES OF TRAJECTORIES EXTRACTED FROM SKI VIDEOS, USING THE LEAVE-ONE-OUT CROSS VALIDATION TECHNIQUE.

\section{Results on the detection of unexpected events}

Experiments on several real videos for the detection of unexpected events using QHMMs were also conducted. For the Formula One race video, the developed QHMM method was able to detect unexpected events such as accidents or cars veering off the track (revealed by an abnormal trajectory shape) and intervention of the safety car (revealed by a quite different speed while the global trajectory shape remains unchanged) For the skiing competition, the method was able to detect falls of skiers. These results show the relevance of the $\dot{\gamma}$ feature to account for both the shape of the trajectory and the speed evolution.

Fig. 11 and Fig. 12 respectively show three Formula One video sequences and two Alpine skiing race video sequences. In each case, the first one belongs to a regular event class while the others are examples of unexpected events. The criterion described in subsection V-B allowed us to correctly detect the unexpected events in all the processed examples. In Table I and IV are supplied, for several unexpected events, the $R_{i}+\sigma_{i}$ criterion values and the distance between the trajectory detected as unexpected event and the considered classes $C_{i}$ (presented in Fig. 8 and 10). Hence, the QHMMbased framework can be straightforwardly and successfully exploited for detecting unexpected events in videos.

\begin{tabular}{|c|c|c|c|c|c|c|}
\hline & Class 1 & Class 2 & Class 3 & Class 4 & Class 5 & Status \\
\hline$R_{i}+\sigma_{i}$ & 0.1315 & 0.0428 & 0.0392 & 0.1442 & 0.0454 & \\
\hline Fall of a skier & 0.1723 & 0.1224 & 0.0715 & 0.6086 & 0.3258 & detected \\
\hline
\end{tabular}

TABLE IV

DETECTION THRESHOLD ARE SUPPLIED IN THE SECOND ROW FOR THE CLASSES OF SKI TRAJECTORIES USED IN THE DETECTION TASK OF UNEXPECTED EVENTS. THE FOLLOWING ROW CONTAINS THE DISTANCE BETWEEN THE EVENT "ACCIDENT" TRAJECTORY AND THE REGULAR CLASSES.

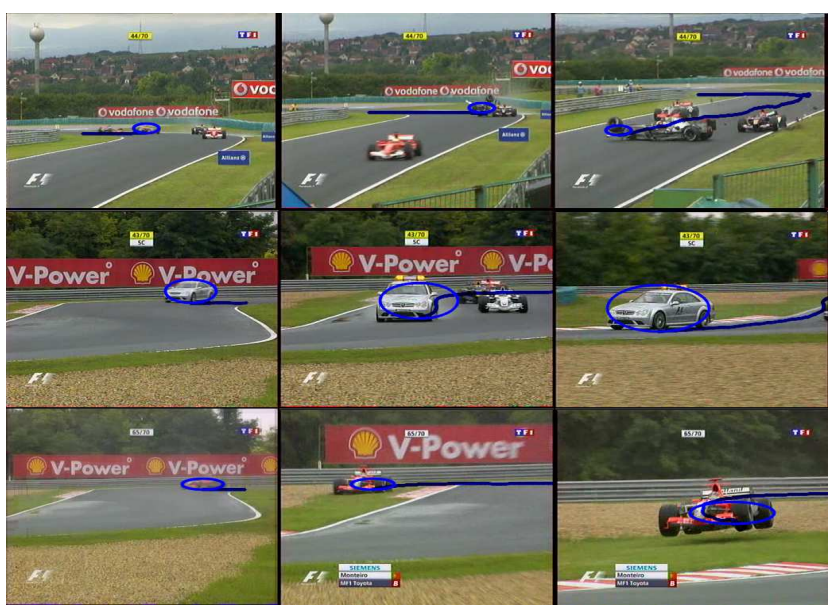

Fig. 11. Images from Formula One race video shots. Each row presents an example of unexpected event (accident, safety car appearance and car driving off the track). The trajectories are overprinted on the images.

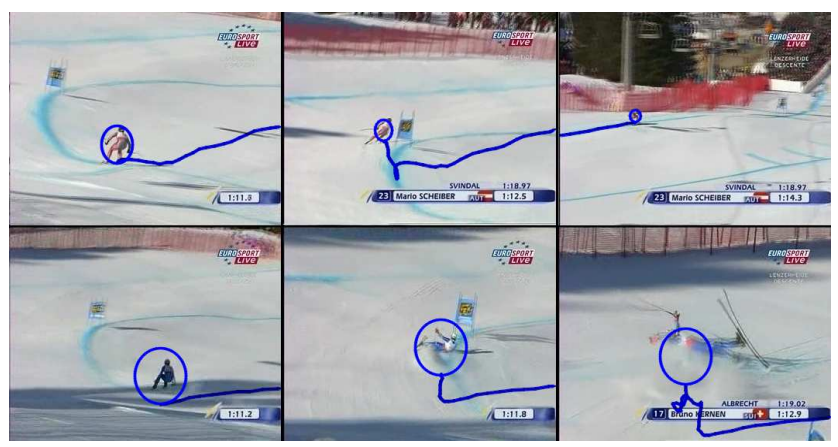

Fig. 12. Images from Alpine skiing competition video shots acquired by the same camera. Trajectories are overprinted on the images. Top row: example of regular class. Bottom row: example of unexpected event (fall of a skier).

\section{CONCLUSION}

A statistical trajectory-based HMM framework for video content understanding was proposed, automatic, general and flexible enough to solve challenging tasks: recognition of events corresponding to learned classes of dynamic video contents and detection of unexpected events. Appropriate local trajectory features invariant to translation, rotation and scale transformations, and that can reliably be computed in presence of noise, have been introduced. Efficient statisticallybased parameter estimations methods were also proposed. An important set of comparative experiments on real videos (sport TV programs) with classification ground truth has been conducted and showed that the proposed method supplies accurate results and offers better performances than other approaches such as histogram comparison, HMM/GMM modeling and SVM classification. Extensions of this work will investigate the representation of activities in videos using hierarchical modeling of interacting space-time groups of trajectories. 


\section{REFERENCES}

[1] N. Anjum, and A. Cavallaro. Unsupervised fuzzy clustering for trajectory analysis. Proc. of the IEEE Int. Conf. on Image processing, ICIP'07, San Antonio, US, Sep. 2007.

[2] G. Antonini, and J.P. Thiran. Counting pedestrians in video sequences using trajectory clustering. IEEE Trans. on Circuit and Systems for Video Technology, 16(8):1008-1020, Aug. 2006.

[3] F. I. Bashir, A. A. Khokhar, and D. Schonfeld. View invariant motion trajectory-based activity classification and recognition. Multimedia Syst., 12(1):45-54, 2006.

[4] F. I. Bashir, A. A. Khokhar, and D. Schonfeld. Real-time motion trajectory-based indexing and retrieval of video sequences. IEEE Trans. on Multimedia, 9(1):58-65, 2007.

[5] F. I. Bashir, A. A. Khokhar, and D. Schonfeld. Object trajectory-based activity classification and recognition using Hidden Markov Models. IEEE Trans. on Image Proc., 16(7):1912-1919, 2007.

[6] O. Boiman, and M. Irani. Detecting irregularities in images and in video. Proc. of the IEEE Int. Conf. on Computer Vision, ICCV'05, Beijing, China, Vol.1, pages 462-469, Oct. 2005.

[7] C. Burges. A tutorial on support vector machines for pattern recognition. Data Mining and Knowledge Discovery, Springer, 2:121-167, 1998.

[8] D. Buzan, S. Sclaroff, and G. Kollios. Extraction and clustering of motion trajectories in video. Proc. of the IEEE Int. Conf. Pattern Recognition, ICPR'04, pages 521-524, Cambridge, UK, Aug. 2004.

[9] S-F. Chang, W. Chen, H J. Meng, H. Sundaram, and D. Zhong. A fully automated content-based video search engine supporting spatiotemporal queries. IEEE Trans. on Circuits and Systems for Video Technology, 8(5):602-615, Sep. 1998.

[10] M. T. Chan, A. Hoogs, J. Schmiederer, and M. Peterson. Detecting rare events in video using semantic primitives with HMM. Proc. of the IEEE Int. Conf. on Pattern Recognition, ICPR'04,pages 150-154, Cambridge, UK, Aug. 2004.

[11] M. T. Chan, A. Hoogs, R. Bhotika, and A. Perera. Joint recognition of complex events and track matching. Proc of the IEEE Conf. on Comp. Vis. and Patt. Rec.,CVPR'06, pages 694-699, New York, Jun. 2006.

[12] R. Fablet, and P. Bouthemy. Motion recognition using non parametric image motion models estimated from temporal and multiscale cooccurrence statistics. IEEE Trans. on Pattern Analysis and Machine Intelligence, 25(12):1619-1624, Dec. 2003.

[13] H. Fashandi, and A.M.E. Moghaddam. A new invariant similarity measure for trajectories. Proc. of the IEEE Int. Symp. on Computational Intelligence in Robotics and Automation, CIRA'05,pages 631-634, Espoo, Finland, June 05.

[14] W. Feller. An Introduction to Probability Theory and Its Applications, Vol. 2, 3rd ed, Wiley, New York, 1971.

[15] J. Ford and J. Moore. Adaptive estimation of HMM transition probabilities. IEEE Trans. on Signal Processing, 46(5):1374-1385, 1998.

[16] Z. Fu, W. Hu, and T. Tan. Similarity based vehicle trajectory clustering and anomaly detection. Proc. of the IEEE Int. Conf. on Image processing, ICIP'05, Genova, Switzerland, Sep. 2005.

[17] S. Gong and T. Xiang. Recognition of group activities using dynamic probabilistic networks. Proc. of the IEEE Int. Conf. on Computer Vision, ICCV'03, pages 742-749, Nice, France, Oct. 2003.

[18] N. Gengembre, and P. Pérez. Probabilistic color-based multi-object tracking with application to team sports. Technical report, INRIA, RR-6555, May. 2008.

[19] W. Härdle, M. Muller, S. Sperlich, and A. Werwatz. Nonparametric and semiparametric models. Springer, Springer series in statistics, Berlin, Germany, 2004.

[20] A. Hervieu, P. Bouthemy, and J-P. Le Cadre. A HMM-based method for recognizing dynamic video contents from trajectories. Proc. of the IEEE Int. Conf. on Image Proc., ICIP'07, San Antonio, US, Sept. 2007.

[21] A. Hervieu, P. Bouthemy, and J-P. Le Cadre. Video event classification and detection using 2D trajectories. Proc. of the Int. Conf. on Comp. Vis. Theory and Applications, VISAPP'08, Madeira, Portugal, Jan. 2008

[22] T. Hastie, R. Tibshirani, and J. Friedman. The elements of statistical learning. Springer series in statistics, Berlin, Germany, 2001.

[23] S. Hongeng, R. Nevatia, and F. Bremond. Video-based event recognition: Activity representation and probabilistic recognition methods. Computer Vision and Image Understanding, 96(2):129-162, 2003.

[24] W. Hu, X. Xiao, T. Tan, and S. Maybank. Learning activity patterns using fuzzy self-organizing neural network. IEEE Trans. on Systems, Man and Cybernetics - Part B: Cybernetics, 34(3):1618-1626, Jun. 2004.

[25] W. Hu, X. Xiao, Z. Fu, D. Xie, T. Tan, and S. Maybank. A system for learning statistical motion patterns. IEEE Trans. on Pattern Analysis and Machine Intelligence, 28(9):1450-1464, Sep. 2006.
[26] W. Hu, D. Xie, Z. Fu, W. Zheng, and S. Maybank. Semanticbased surveillance video retrieval. IEEE Trans. on Image Processing, 16(4):1168-1181, Apr. 2007

[27] T. Izo. Visual Attention Models for Far-Field Scene Analysis. Ph.D. Thesis, MIT, Cambridge, US, Jun. 2007.

[28] T. Izo, and W.E L. Grimson. Unsupervised modeling of object tracks for fast anomaly detection. Proc. of the IEEE Int. Conf. on Image processing, ICIP'07, San Antonio, US, Sep. 2007.

[29] N. Johnson, and D. Hogg. Learning the distribution of object trajectories for event recognition. Proc. of British Machine Vision Conf., BMVC'95, Birmingham, UK, Jul. 1995.

[30] I N. Junejo, and H. Foroosh. Trajectory Rectification and Path Modeling for Video Surveillance. Proc of the IEEE Int. Conf. on Computer Vision, ICCV'07, Rio de Janeiro, Brazil, Oct. 2007.

[31] S. Khalid, and A. Naftel. Classifying spatiotemporal object trajectories using unsupervised learning of basis function coefficients. ACM int work. on Video Surv. and Sensor Net., VSSN'05, Singapore, Nov. 2005.

[32] A. Kokaram, N. Rea, R. Dahyot, M. Tekalp, P. Bouthemy, P. Gros, and I. Sezan. Browsing sports video (Trends in sports-related indexing and retrieval work). IEEE Signal Processing Mag., 23(2):47-58, Mar. 2006.

[33] X. Li, W. Hu, and W. Hu. A coarse-to-fine strategy for vehicle motion trajectory clustering. Proceedings of the IEEE Int. Conf. on Pattern Recognition, pages 591-594, Hong Kong, Aug. 2006.

[34] J. Lou, Q. Liu, T. Tan, and W. Hu. Semantic interpretation of object activities in a surveillance system. Proc. of the IEEE Int. Conf. on Patt. Rec., ICPR'02, pages 777-780, Quebec, Canada, Aug. 2002.

[35] D. Makris, and T. Ellis. Path detection in video surveillance. Image and Vision Computing, 20(12), pages 895-903, 2002.

[36] D. Makris, and T. Ellis. Learning semantic scene models from observing activity in visual surveillance. IEEE Trans. on Systems, Man and Cybernetics - Part B: Cybernetics, 35(3):397-408, June. 2005.

[37] J. Melo, A. Naftel, A. Bernardino, and J.S. Victor. Retrieval of vehicle trajectories and estimation of lane geometry using non-stationary traffic surveillance cameras. Proc of the IEEE Int. Conf. on Adv. Concepts for Intelligent Vision Syst., ACIVS'04, Brussels, Belgium, Aug-Sept. 2004.

[38] A. Naftel, and S. Khalid. Classifying spatiotemporal object trajectories using unsupervised learning in the coefficient feature space. Multimedia Syst., 12(3):227-238, 2006.

[39] N. M. Oliver, B. Rosario, and A. P. Pentland. A Bayesian computer vision system for modeling Human Interactions. IEEE Trans. Pattern Analysis and Machine Intelligence, 22(8):831-843, Aug. 2000.

[40] P. Perez, C. Hue, J. Vermaak, and M. Gangnet. Color-based probabilistic tracking. Proc. Europ. Conf. Computer Vision, ECCV'02, Copenhaguen, Denmark, Jun. 2002.

[41] C. Piciarelli, G. L. Foresti, and L. Snidaro. Trajectory clustering and its applications for video surveillance. Proc. of the IEEE Int. Conf. on Advanced Video and Signal based Surveillance, AVSS'05, pages 40-45, Como, Italy, Sept. 2005.

[42] G. Piriou, P. Bouthemy, and J.-F. Yao. Recognition of dynamic video contents with global probabilistic models of visual motion. IEEE Trans. on Image Processing, 15(11):3417-3430, Nov. 2006.

[43] F. Porikli. Trajectory distance metric using hidden Markov model based representation. Workshop on Performance Evaluation for Tracking. and Surveillance (PETS), Pragia, Czech Republic, May 2004.

[44] L. Rabiner. A tutorial on hidden Markov models and selected applications in speech recognition. Proc. IEEE, 77(2):257-285, 1989.

[45] P. Remagnino, A. I. Shihab, and G. A. Jones. Distributed intelligence for multi-camera visual surveillance. Pattern Recognition, 37(4):675-689, Apr. 2004.

[46] C. Stauffer, and W. E. L. Grimson. Learning patterns of activity using real-time tracking. IEEE Trans. Pattern Analysis and Machine Intelligence, 22(8):747-757, Aug. 2000.

[47] M. Vlachos, G. Kollios, and D. Gunopulos. Discovering similar multidimensional trajectories. Proc. of the IEEE Int. Conf. on Data Engineering, ICDE'02, San Jose, US, Feb 2002.

[48] X. Wang, K. Tieu, and E. Grimson. Learning semantic scene models by trajectory analysis. Proc. Europ. Conf. Computer Vision, ECCV'06, Graz, Austria, May 2006.

[49] Z. Zhang, K. Huang, and T. Tan. Comparison of similarity measures for trajectory clustering in outdoor surveillance scenes. Proc. of the IEEE Int. Conf. on Pattern Recognition, ICPR'07, pages 1135-1138, Hong Kong, Aug. 2006.

[50] Z. Zhang, K. Huang, T. Tan, and L. Wang. Trajectory series analysis based event rule induction for visual surveillance. Proc. of the IEEE Conf. on Computer Vision and Pattern Recognition, CVPR'07, Minneapolis, US, Jun. 2007. 